\title{
Recent developments in herbal drug standard in India
}

\begin{abstract}
Herbal medicines are regarded as the oldest and most extensively used medicinal system. The demand for herbal products both in the form of medicines and dietary supplements are gaining rapid momentum throughout the world. This fast expansion of the global market for herbal products have raised serious concerns among health authorities, pharmaceutical industries and consumers regarding the safety and quality of medicinal plant materials and finished herbal formulations. Like any other chemical pharmaceutical product, quality, safety and efficacy are utmost important for herbal products as well. However, lack of evidence based practice, consistent quality approach and scanty research on traditional treatment methods are few of the challenges which the herbal drug market is continuously facing. ${ }^{1}$ Herbal products used as dietary supplements or nutraceuticals are also facing the problems of adverse events due to lack of regulation, weak quality control systems and loose distribution channels. India, the second largest exporter of medicinal plants, is a major hub for different traditional herbal medicinal products worth USD 3 billion domestic market and is also constantly facing the challenges associated with the quality and safety. With more than 8000 manufacturing units of which majority being small or medium size firms, Indian herbal industry is grappling with the issues of standardization of raw materials and finished formulations, evidence based practices for AYUSH(Ayurveda, Unani, Siddha and Homoeopathy) medicines. ${ }^{1,2}$
\end{abstract}

Keywords: pulmonary, lung function, respiratory muscles power, asthma
Volume 12 Issue 5 - 2019

\author{
Niharika Sahoo Bhattacharya \\ Assistant Professor, Rajiv Gandhi School of Intellectual Property \\ Law, India \\ Correspondence: Niharika Sahoo Bhattacharya, Assistant \\ Professor, Rajiv Gandhi School of Intellectual Property Law, IIT \\ Kharagpur,West Bengal, 72I302, India, \\ Email contact.niharika@gmail.com, niharika@rgsoipl.iitkgp.ac.in
}

Received: October 06, 2019 | Published: October 18, 2019

\section{Herbal drug standardization}

Inherent complex nature of plants makes the standardization of herbal medicines a difficult task. The therapeutic actions of diverse secondary metabolites largely depend on age, genetic factors and geographical location of the plant species ${ }^{3}$. The same plant species may show substantial different phytochemical leading to variable pharmacological actions. The heavy metal contamination and adulteration due to microorganisms and pesticides furthers creates difficulties in achieving uniform standards of herbal medicines worldwide. The raw material harvesting seasons, procedure, storage and processing of the same are critical in maintaining the quality of the herbal medicines ${ }^{4}$. Another major issue with Indian ASU medicines is the polyherbal nature of the medicine, which makes them difficult for standardization. Non-availability of post-harvest facilities at primary level, lack of testing laboratories at the raw material production site, lack of product traceability are few of the important concerns from quality point of view.

Various chromatographic, sequencing technologies including the 'omic' technologies are proving to be effective tools for standardization of herbal drugs. The latest herbal genomics approach provides an effective platform to support the chemical and biological analyses of complex herbal products that may contain more than one active component. It may be used to understand the quality of traditional medicines and for molecular herb identification ${ }^{5}$. However, the high cost associated with the same remains a challenge for the small scale manufacturers.
To mitigate the challenges associated with raw material collection implementation of good agricultural practices (GAPs) at the point of cultivation of medicinal plants and good manufacturing practices (GMPs) during the process of manufacture and packaging of finished herbal products, as well as post-marketing quality assurance surveillance are essential for improving the quality of herbal medicines. The following table lists the available guidelines for ASU drugs.

\section{Quality related guidelines for ASU drugs}

Quality control methods for medicinal plant materials(WHO, 1998), ${ }^{6}$

2 Quality control methods for herbal materials (WHO 20II)

3 Laboratory Manual for the Analysis of Ayurveda and Siddha formulations (CCRAS, 20I0) ${ }^{8}$

4

Quality Control Manual for Ayurvedic, Siddha \& Unani Medicine, Pharmacopoeial Laboratory for Indian Medicine (PLIM, 2008) ${ }^{9}$

5 Protocol for Testing of ASU Medicines, (PLIM, 2007) ${ }^{10}$

General Guidelines For Drug Development Of Ayurvedic Formulations (CCRAS, 2016)"

$7 \quad$ ASU Pharmacopoeias ${ }^{12}$ 


\section{Safety and Toxicity Study guideline for ASU drugs}

\begin{tabular}{ll}
\hline I & $\begin{array}{l}\text { General Methodologies \& Research evaluation Traditional } \\
\text { Medicines (WHO, 2000) }\end{array}$ \\
2 & OECD guidelines, 200I ${ }^{14}$ \\
3 & Schedule Y of Drug and Cosmetic Act, $1945^{15}$ \\
4 & GCP Guidelines for ASU Medicines, 20I3, Ministry of AYUSH ${ }^{16}$ \\
5 & $\begin{array}{l}\text { General Guidelines of Safety/Toxicity Evaluation of Ayurvedic } \\
\text { Formulations(CCRAS, 20 I6) }\end{array}$
\end{tabular}

Good Clinical practice guideline for ASU drugs

I General Guidelines for Clinical Evaluation of Ayurvedic Interventions(CCRAS, 2016) ${ }^{18}$

Also under the aegis of Ministry of AYUSH, the research wing Central Council for Research in Ayurvedic Sciences (CCRAS) has been involved in standardization and safety studies of various Ayurvedic formulations. So far 160 drugs were screened for various safety/toxicity of which 54 are Single drugs, 31 are Ayurvedic Formulations and 75 are Coded drugs. To generate scientific evidence on safety of most commonly used classical Ayurvedic herbo-mineral formulations, biological screening of nearly 389 drugs has been completed till 2018.

\section{Evidence based practice}

The Indian traditional medicine has been historically based on ancient texts, experts, clinical experiences. The belief that herbal medicines are natural products and free from side effects has helped the traditional medicinal system to gain peoples' acceptance. The cost effectiveness and comparative safety, holistic use and disenchantment with chemical drugs have further increased the acceptability of medicine of traditional Indian systems: Ayurveda, Siddha and Unani (ASU), which are explored for providing therapeutic solutions to the emerging health problems. However, there has been persistent and increasing demand of documented proof of clinical safety and efficacy of ASU medicines.

Going by statutory provisions, there are two types of ASU medicine, one being the classical medicine as mentioned in the authoritative books of Ayurveda, Siddha and Unani Medicine and the other category of Patent or Proprietary medicines. The classical medicines are manufactured and named in accordance with the formulations described in the authoritative texts, and do not require further clinical evidences. But, the herbal medicines which contain ingredients referred in the formulations of authoritative texts but the inventor's intellectual intervention or innovation made them different from the classical medicine requires further validation. Validation of safety and efficacy using scientific and evidence-based methodologies not only solves statutory requirement but also helps in gaining universal acceptability. It further adds to confidence of practitioners and satisfaction of end users in the products.

With the introduction of Drugs \& Cosmetics Rule 158 B since 2010 , the requirement of proof of effectiveness for licensing of patent or proprietary ASU medicine has necessitated the development of present guidelines of Good Clinical Practice. These guidelines include standards of how clinical trials should be conducted; and define the roles and responsibilities of clinical trial sponsors, clinical research investigators, monitors etc. The objective is to inculcate the culture of conducting ASU intervention-based clinical studies in the country in accordance with requisite scientific standards and appropriately designed methodologies. It also aims that results and findings of clinical trials are properly recorded, analyzed and reported. Emphasis is given so that the clinical research generates quality data, acceptable to regulatory authorities for product registration or marketing approval especially for non-classical or non-generic formulations.

Another step towards ensuring herbal drug quality was the mandatory mentioning of the date of expiry ASU medicines on the label of container or package of respective medicines. Every person applying for licence or renewal of licence for the manufacturing of ASU medicines shall submit to the State Licensing Authority scientific data based shelf life or date of expiry of the medicine based on the real time stability studies of medicines in accordance with the guidelines prescribed in the Ayurvedic Pharmacopoeia of India.

\section{ASU as nutraceuticals}

At present various kinds of ASU products licensed in the country are being sold as neutraceuticals or food supplements (Balya/Poshak). They are generally considered free from any adverse effect and safe to consume. The Drugs \& Cosmetics Act does not define these ASU products which fall under category Neutraceutical or food supplement. And there is no regulation existing regarding said ASU products under above said category. As per recommendation of Ayurveda, Siddha and Unani Drug Technical Board, the Amendment to Rule 158(B) regarding guidelines for issue of license in respect of ASU drugs have been carried out and Draft Notification has been issued on 2010. However, still there is no clear regulation for the same.

\section{Conclusion}

A scientific justification on quality, safety and efficacy of the traditional herbal medicinal product is essential for both the consumers and regulators. Also, this will promote the integration of traditional system of medicine with the mainstream treatment method with chemical pharmaceuticals.

\section{Acknowledgments}

None.

\section{Conflicts of interest}

The authors declare there are no conflicts of interest related to the article.

\section{References}

1. Sahoo N, Manchikanti P, Dey S. Herbal drugs: Standards and regulation. Fitoterapia. 2010;81(6):462-471.

2. Bhatia N. Ayurveda Industry - Market Size, Strength and Way Forward. India: Confederation of Indian Industry; 2018.

3. Firenzuoli F, Gori L. Herbal medicine today: clinical and research issues. Evid Based Complement Alternat Med. 2007;4:37-40.

4. Chikezie PC, Ojiako OA. Herbal Medicine: Yesterday, Today and Tomorrow. Altern Integr Med. 2015;4:195.

5. Chen. Herbal genomics: Examining the biology of traditional medicines. The Art and Science of Traditional Medicine Part 2: Multidisciplinary 
Approaches for Studying Traditional Medicine Science. Science. 2015;347(6219 Suppl): S27-S29.

6. World Health Organization (WHO). Quality control methods for medicinal plant materials. Geneva: WHO; 1998. p.1-127.

7. World Health Organization (WHO). Quality control methods for herbal materials. Geneva: WHO; 2011. p. 1-187.

8. Lavekar, Padhia EE, Pant P, et al. Laboratory Manual for the Analysis of Ayurveda and Siddha formulations. India: Central Council for Research in Ayurvedic Sciences. Ministry of AYUSH; 2010.

9. Quality Control Manual for Ayurvedic, Siddha \& Unani Medicine. India: Pharmacopoeial Laboratory for Indian Medicine. 2008.

10. Protocol for Testing of ASU Medicines. India: Central Council for Research in Ayurvedic Sciences. Ministry of AYUSH; 2007. p. 1-200.

11. General Guidelines For Drug Development Of Ayurvedic Formulations (CCRAS, 2016). India: Central Council for Research in Ayurvedic Sciences. Ministry of AYUSH; 2016. p. 1-123.
12. Pharmacopoeia Commission for Indian Medicine \& Homoeopathy.

13. World Health Organization (WHO). General guidelines for Methodologies on Research and evaluation of Traditional Medicines. Geneva: WHO; 2000. p. 1-80.

14. OECD guidelines for testing of chemicals: Acute oral toxicity-acute toxic class method. 2001

15. Schedule Y of Drug and Cosmetic Act of India.1945.

16. GCP Guidelines for ASU Medicines. India: Ministry of AYUSH; 2013. p. $1-114$.

17. General Guidelines of Safety/Toxicity Evaluation of Ayurvedic Formulations (CCRAS, 2016). India: Central Council for Research in Ayurvedic Sciences. Ministry of AYUSH; 2018. p. 1-94.

18. General Guidelines for Clinical Evaluation of Ayurvedic Interventions (CCRAS, 2016). India: Central Council for Research in Ayurvedic Sciences. Ministry of AYUSH; 2018. p. 1-123. 